\title{
Characteristics of Bonesetting Therapy in Chinese Mongolian Osteopathy
}

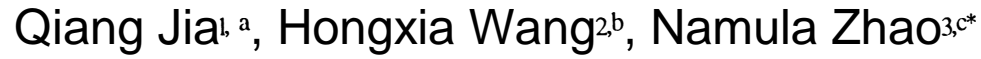 \\ ${ }^{1}$ Beijing Meigin Vision Technology Co., Ltd, Beijing, China \\ ${ }^{2}$ DongXun Technology (Beijing) Co., Ltd, Beijing, China \\ ${ }^{3}$ Research Center for The Traditional Mongolian Medicine, Inner Mongolia University for the Nationalities, \\ Tongliao, China \\ aopensec@163.net, bwanghx@dascom.cn, ${ }^{\mathrm{c} z n m l @ 263 . n e t ~}$
}

Key Words: osteopathy of traditional Mongolian medicine; fracture; feature

\begin{abstract}
Objective: Chinese Mongolian osteopathy has been passed down from ancient times and includes unique practices and favorable efficacy. In this study, we investigate the characteristics of individualized Chinese Mongolian osteopathy, in order to provide new principle and methods for the treatment of bone fracture. Method: With a view to provide a vital link between nature and humans, the four stages of Chinese Mongolian osteopathy focus on the unity of the mind and body, the limbs and body organs, the body and its functions, and humans and nature. Results: We discuss the merits of individualized osteopathy in terms of the underlying concepts, and evaluate the approaches and principles of traditional medicine, as well as biomechanics. Conclusions: The idea, manipulation and norm on reposition and heal of fracture in osteopathy of traditional Mongolian medicine in China astonishingly coincide with the theory on micro-mechanism of "stress adaptation - function adaptation" and with the principle on "structural continuity - functional wholeness" in repair and heal of fracture in modern orthopedics. This is the radical reason why osteopathy of traditional Mongolia medicine in China has been used so far and the direction in which the concept of osteopathy will develop wholly, dynamically and functionally.
\end{abstract}

\section{INTRODUCTION}

Chinese Mongolian osteopathy, which is widely practiced in the Mongolian grasslands of China, has existed since ancient times. It is unique not only because of the treatments used and healing effects, but also because of its continued use since the first descendant of Bao's traditional osteopathic practitioner, Naran Abai (1790-1875). According to the literature[1-3], the foundation of Chinese Mongolian osteopathy is dependent on the unique Mongolian ecological environment, habits and customs, as well as historical background and culture. Ultimately, modern-day Chinese Mongolian osteopathy represents culmination of extensive knowledge on the treatment of bone fracture. This approach is built upon the principles of life sciences that conform to natural laws.

Although modern osteopathy turned from a strong static fixation concept in the 1950s to an elastic dynamic fixation idea in the 1980s, the deleterious effects of static fixation have not been appreciated until recent years. Chinese Mongolian osteopathic practices are based on hommization, actualized and integrated therapeutic characteristics of reduction, fixation and healing, whereas the modern science of osteopathy has 
not extricated itself from the abyss caused by the lack of individualization, actualization and integration. Considering the historical background and longevity of Chinese Mongolian osteopathy, introducing the concepts held in these traditional practices may help to improve current treatments for bone fractures [3, 4].

\section{TREATMENT METHODOLOGY}

Chinese Mongolian osteopathy follows a four-step process[5] intended to reinforce the link between nature and humans, including the unity of mind and body, the link between limbs and body organs, and between the body and its functions. Chinese Mongolian osteopathy, as well as long-standing clinical practices $[2,4]$ involves fracture treatmentand encompasses traditional medicine, as well as the principles and methods of biomechanics: ( in Figure1).

$$
\text { (Condition 1) (Target 1) (Condition 2) (Target 2) }
$$

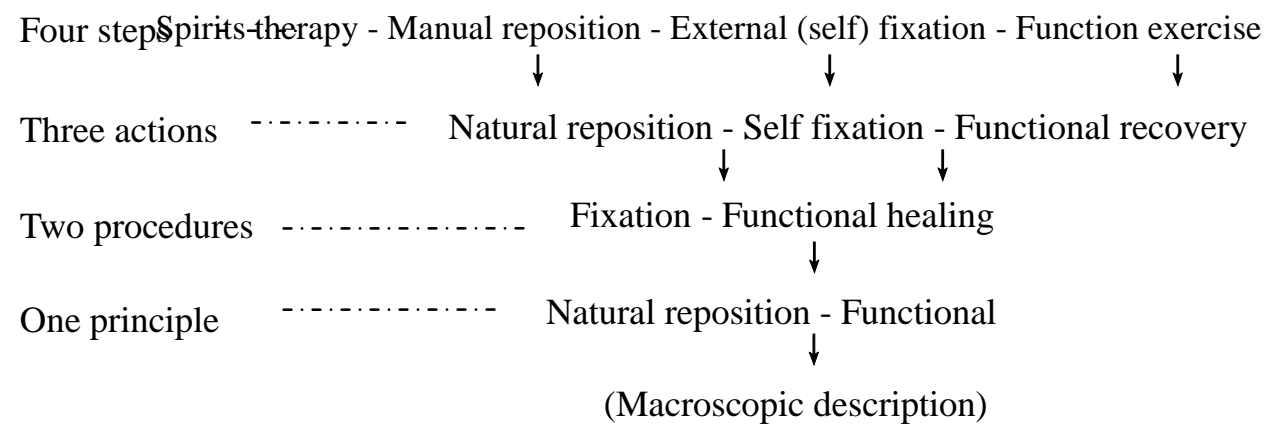

Figure1. The concept of Mongolian osteopathy

Based on the established in the Chinese Mongolian osteopathy follows a four-step process[5] intended to reinforce the link between nature and humans, including the unity of mind and body, the link between limbs and body organs, and between the body and its functions.

So, the four steps in Chinese Mongolian osteopathy focus on three outcomes, namely reduction, fixation and healing. Each outcome requires fracture treatment and has specific characteristics.

\section{RESULTS AND DISCUSSION}

Dynamic reduction, natural fixation and self-healing of bone fractures: characteristics of fracture treatment Chinese Mongolian osteopathy includes a treatment technique called 'spray spirits and stroke fracture', which is a type of physiological and psychological naturopathy that is performed before attempting fracture reduction. Unique maneuvering integrates the 'spirits therapy' and manipulates the fracture into 'dynamic reduction', one of the fracture treatment therapeutic features of Chinese Mongolian osteopathy[2,5,6]. To achieve a good environment for manual repositioning, surgeons spray high-speed atomized spirits that fall around the bone fracture after 'viewing, touching, and judging' the bone and reading the X-ray film. Meanwhile, the practitioner strokes the fracture. Studies have shown that high-speed atomized spirits falling around the bone fracture causes the following effects [7-9]:

1) High-speed atomized spirits decrease the local pressure and temperature, causing rapid cooling, which reduces swelling, fever and pain. 
2) As soon as the 'spirits factor' permeates the muscles and tendons at the end of the bone fracture, it activates and interconnects muscles and tendons, and encourages the flow of blood and vital energy. Then, through nerve pathways, these effects are transmitted to the higher nervous systems to stimulate individual' s desire for self-reduction and self-healing.

3) The sweet sounding whistle caused by the high-speed atomized spirits also exerts psychological effects on the patient. Indeed, clinical practice indicates that the treatment 'spray spirits and stroke fracture' usually encourages a self-healing state in the patients.

Chinese Mongolian osteopathy can be summed up in 10 reduction skills that have been developed and refined over many years. All of these 10 skills meet the modern biomechanic criterion of 'force to force' [10]. Chinese Mongolian osteopathy provides complete repositioning through a 'force to force' maneuver when a patient reaches the optimal physiological and psychological condition.

Based on the view of a vital link between nature and humans, including the unity of mind and body, repositioning in Chinese Mongolian osteopathy is a natural dynamic therapy using 'force-to-force' maneuvers and capitalizes on the time when patients reach an optimal physiological and psychological condition. It also includes characteristics including natural, self-healing and dynamic reduction of bone fractures, and causes less pain, precise reduction, fast healing and low treatment cost compared with conventional therapy.

Biological macroscopic characteristics of fracture treatment for bone fractures: 'unobstructed, natural fixation, closely and openly, self-healing Fixation represents a nexus between repositioning and healing. Chinese Mongolian osteopathy integrates fixation using small splints and physiological and psychological self-fixation. Self-fixation is the second most important feature of Chinese Mongolian osteopathy $[2,11,12]$. As before, self-fixation is based on the view of a vital link between nature and humans. Self-fixation integrates the mosaic of forces at the end of the bone fracture by exploiting the natural biological splints (i.e., self-fixation) provided by muscles, tendons and skin, as well as the application of thin light splints placed according to the location of the bone fracture and movement. Three splints are used to bind the bone fracture at the midpoint and far ends of the bandages. As shown in Figure 2, self-fixation and external fixation can be represented by a dynamic stable fixation system of the bone fracture [2, 13]. This figure shows that self-fixation exploits various features to provide dynamic stable fixation of the bone fracture (Fig. 2: the t-axis shows the surface of the fracture, then-axis shows the normal vector to the t-axis).

Chinese Mongolian osteopathy attaches great importance to the self-healing instinct of humans and therefore plays a leading role in treatment. For incomplete fracture, Chinese Mongolian osteopathy practitioners do not use external fixation, whereas for a complete fracture, practitioners advocate self-fixation and correct use of small splints.

\section{1) Geometric dynamic fixation of the bone fracture}




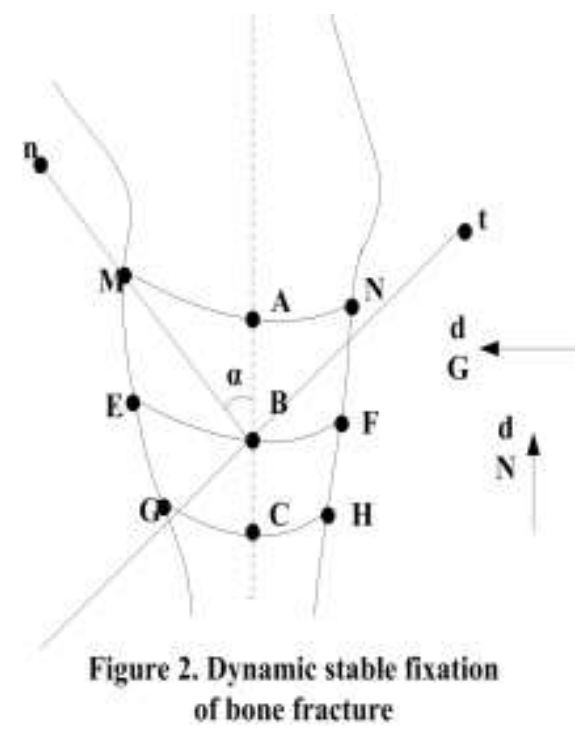

As shown in Figure 2, each splint is fixed at the middle and ends of the bandages along the diaphysis (in Figure2), and maintains stability according to the principle 'two points define a line'. Along the direction vertical to the diaphysis, each splint is fixed at the near end, middle and far end (in Figure 2) and maintain their stabilities according to the principle 'three points define a plane'. Therefore, the three-dimensional stability of the end of the bone fracture is maintained[2,13]. Because of the flexibility of the splints and a moderate degree of tightness (elasticity) of the bandage, the fixation exploits the effects of geometric dynamic structural stability, and encourages natural healing.

\section{2) Macroscopic biological effects of the dynamic structural stability of bone fractures}

If $\tau=$ the axis along the plane of the bone fracture, then the equation for the mechanical equilibrium at the end of the bone fracture relative to a cross section is[2, 13$]$ :

$$
\sin \alpha d N-\cos \alpha d G \leq K_{\text {static }}(\cos \alpha d N+\sin \alpha d G)
$$

When $\alpha$ is a constant,

$$
N \leq \frac{K_{\text {static }} \sin \alpha+\cos \alpha}{\sin \alpha-K_{\text {static }} \cos \alpha} G
$$

This is a sufficient and necessary condition to achieve equilibrium of forces at the end of a bone fracture relative to a cross section. Here $\mathrm{dN}$ is the axial force of the diaphysis, $\mathrm{dG}$ is the binding force of the bandage (including the forces $\mathrm{dGx}$ and $\mathrm{dGy}$ for reactive and active movements, respectively), and $\alpha$ is the angle between the normal cross section of the bone fracture and the diaphysis.

When the value of kstatic (the coefficient of the resistance force of embedding) is sufficiently large, and assuming the binding force $\mathrm{G}$ is chosen according to the axial force $\mathrm{N}$ of the diaphysis, this satisfies the 
necessary and sufficient condition to achieve equilibrium of the forces encountered at the end of the bone fracture. Therefore, this approach can maintain an equilibrium of the forces at the end of the bone fracture.

\section{3 ) Biological macroscopic characteristics of kinematic dynamic fixation}

Self-fixation using small splints is based on the geometric dynamic stability and mechanical dynamic equilibrium, usually with the aid of relatively static fixation. Self-fixation can maintain the kinematic stability of the end of the bone fracture and stimulate the physiological processes necessary for bone fracture healing. The fixation condition can also maintain kinematic stability of the end of the bone fracture [2,5,11]. At the end of the bone fracture, the dynamic properties are relative to the static properties resulting in unitary static and dynamic forces. Therefore, the macroscopic biological effects used to achieve equilibrium help promote the natural conditions necessary for bone fracture healing.

4) Characteristics of the stability between osteoblasts and osteoclasts during dynamic fixation of bone fractures

For self-fixation using small splints, the physiological stress at the end of a fractured bone can be expressed as[2, 11, 14]:

$$
d \sigma=\frac{d G_{x}+d G_{g}}{\frac{A_{0}}{\cos \alpha}} \sin \alpha+\frac{d N}{\frac{A_{0}}{\cos \alpha}} \cos \alpha
$$

when $\alpha$ is a constant,

$$
\frac{d G_{x}}{2 A_{0}} \sin 2 \alpha+\frac{1}{2 A_{0}}\left[G_{g} \sin 2 \alpha+N(1+\cos 2 \alpha)\right]
$$

Where $A_{0}$ is the cross section of the diaphysis, the formula represents a constant physiological stress without functional activity. Accordingly friction force can be increased and shearing force reduced, thus promoting mutual compact embedding at cross-sections of broken bone, shortening the distance between functional osteoblasts and osteoclasts, to accelerate fracture healing. The function represents a discontinuous physiological stress that varies with functional activity. It is a vital natural force that promotes osteoblast activity and osteogenesis. These physiological stresses can maintain the functional stability of a bone fracture. At the end of a bone fracture, physiological stresses exhibit constant or discontinuous stimulation. This implies a macroscopic biological effect of an equilibrium between osteoblasts and osteoclasts in functional bone fracture healing.

\section{5) Characteristics of psychological factors experienced during dynamic fixation of bone fractures}

The ingenious musculoskeletal allows the human body to maintain various postures and perform complex movements. After a bone fracture, under physiological and psychological instructions from the nervous system, the human body can direct healing energy to the fracture location through self control. The human body is also capable of restoration. Therefore, the bone fracture not only undergoes mechanical 
self-fixing but also has physiological and psychological self-fixing. Chinese Mongolian osteopathy protects and stimulates the self-control and self-healing latent functions of the human body, and maintains the psychological stability of patients with a bone fracture[5,11]. Because the ends of the fractured bone are within a person in a natural setting, in a closed or open condition, the macroscopic biological effects include psychological factors that protect and maintain the natural bone fracture healing.

Based on the concept of a link between nature and humans, fixation in Chinese Mongolian osteopathy integrates external fixation using small splints and self-fixation facilitated by physiological and psychological factors, to achieve unobstructed and natural fixation of bone. Self-fixation in Chinese Mongolian osteopathy includes the characteristics of fracture treatment for natural fixation and self-healing. Healing results in an unobstructed bone fracture without scarring or wounding, and is free from infection.

\section{Characteristics of fracture treatment for natural self-healing of bone fractures}

Chinese Mongolian osteopathy integrates natural fixation of bone fractures and early exercise as the third feature of its unique treatment plan $[2,11,15]$. In self-fixation, using small splints for mechanical dynamic fixation, the patient can perform exercises early in treatment and can be exposed to normal physiological stresses at an appropriate time [11, 14].

$\frac{G_{x}}{2 A_{0}} \sin 2 \alpha$ describes the end of a bone fracture and discontinuous physiological stresses ${ }^{[9,5]}$ $\frac{1}{2 A_{0}}\left[G_{g} \sin 2 \alpha+N(1+\cos 2 \alpha)\right]$. Therapy integrates early progress and, step-by-step achieves the best recovery results with a balance between static and dynamic fixation, as well as a balance between osteoblast and osteoclast activities.

Based on the link between nature and humans described above, Chinese Mongolian osteopathy integrates natural fixation of bone fractures and early exercise, achieving functional recovery of fractures restoring the links between the limb and body organs, the body and its functions, and between humans and nature.

\section{CONCLUSIONS}

Chinese Mongolian osteopathy originated from a population living in the grasslands of Mongolia, and is firmly established on the concept of a link between nature and humans, which encompasses unity of the mind and body. Accordingly, Mongolian osteopathy is a natural, ecological and woundless therapy[1, 2, 3].

The unique ideas and treatment practices for bone fracture dynamic reduction and functional healing employed in Chinese Mongolian osteopathy is consistent with the modern concept of 'irritation adaptability-functional adaptability', which has been proposed for the restoration and healing of bone fractures in modern osteopathy[2, 14,15]. Chinese Mongolian osteopathy is fundamentally important because of its ancient history and continuation to modern times. The tenets of Chinese Mongolian 
osteopathy are also appropriate to guide future developments of modern osteopathy, which is turning its attention to personalized, actualized and integrated therapy.

\section{REFERENCES}

[1]ZHAO Namula, ER Dunchaolu, A Gula, JI Rigala, BAO Haijin. Thought on the projective research on Mongolian traditional osteopathy. Journal of Medicine \& Pharmacy of Chinese Minorities [in Chinese], 2002; 8(4):47-48.

[2]ZHAO Namula. New Theory in Biomechanics in Chinese Mongolian Osteopathy, 1st edition, Inner Mongolian People's Press[in Chinese], 2008: 130-187.

[3]Cooper EL. An Emerging Linkade with Ethnopharmacology. eCAM, 2008; 5(4):365-366.

[4] Pettman E. A History of Manipulative Therapy. JMMT, 2007;15(3) :165-174.

[5]ZHAO Namula, Mei W, HU Dalai, HU Rile, AO Wendagula. Natural therapy's characteristics, original ideas, and techniques of Chinese Mongolian medicine tradition osteopathy. Chinese Journal of Traditional Medicine Traumatology \& Orthopedics[in Chinese], 2009; 17(5):66-67.

[6]Huijbregts PA. A New Model for Orthopedic Manual Therapy Research: Description and Implications. JMMT, 2007; 15(4):197-199.

[7]ZHAO Namula. Biomechanics on spraying and regulating therapy in Mongolian traditional osteopathy. China's Naturopathy [in Chinese], 2001; 9(12):55-56.

[8]Meng H, Zhihua G. Biomechanics on Traumatology. Beijing: People Hygiene Press [in Chinese], 1999:6(10):64,279.

[9]ZHAO Namula, Xueen L, Mei W. Macroscopic model for biological immobilization and its uncovering idea in Chinese Mongolian traditional osteopathy. Chinese Journal of Traumatology, 2009; 12(4):234-237.

[10]ZHAO Namula. Discussion on mathematics-physics property on splint external (automatic) fixation in Mongolian traditional osteopathy. Journal of Medicine \& Pharmacy of Chinese Minorities [in Chinese], 2002; 8(1):23-24. 